\title{
Improving Airways Patency and Ventilation Through Optimal Positive Pressure Identified by Noninvasive Mechanical Ventilation Titration in Mounier-Kuhn Syndrome: Protocol for an Interventional, Open-Label, Single-Arm Clinical Trial
}

Evelise Lima*, MD; Maria Aparecida Miyuki Nakamura", PhD; Pedro Rodrigues Genta ${ }^{*}$ PhD, MD; Ascedio José Rodrigues ${ }^{*}, \mathrm{MD}$; Rodrigo Abensur Athanazio*, PhD, MD; Samia Rached", MD; Eduardo Leite Vieira Costa ${ }^{*}, \mathrm{PhD}$, MD; Rafael Stelmach*, PhD, MD

Pulmonary Division-Heart Institute (InCor), Hospital das Clínicas da Faculdade de Medicina de São Paulo, São Paulo, Brazil

*all authors contributed equally

\section{Corresponding Author:}

Evelise Lima, MD

Pulmonary Division-Heart Institute (InCor)

Hospital das Clínicas da Faculdade de Medicina de São Paulo

Dr. Eneas de Carvalho Aguiar, 44

São Paulo

Brazil

Phone: 551126615612

Email: eveliselima53@gmail.com

\begin{abstract}
Background: Mounier-Kuhn syndrome or congenital tracheobronchomegaly is a rare disease characterized by dilation of the trachea and the main bronchi within the thoracic cavity. The predominant signs and symptoms of the disease include coughing, purulent and abundant expectoration, dyspnea, snoring, wheezing, and recurrent respiratory infection. Symptoms of the disease in some patients are believed to be pathological manifestations arising due to resident tracheobronchomalacia. Although treatment options used for the management of this disease include inhaled bronchodilators, corticosteroids, and hypertonic solution, there is no consensus on the treatment. The use of continuous positive airway pressure (CPAP) has been reported as a potential therapeutic option for tracheobronchomalacia, but no prospective studies have demonstrated its efficacy in this condition.
\end{abstract}

Objective: The purpose of this is to identify the presence of tracheobronchomalacia and an optimal CPAP pressure that reduces the tracheobronchial collapse in patients with Mounier-Kuhn syndrome and to analyze the repercussion in pulmonary ventilation. In parallel, we aim to evaluate the prevalence of obstructive sleep apnea/hypopnea syndrome.

Methods: This interventional, open-label, single-arm clinical trial will enroll patients who are diagnosed Mounier-Kuhn syndrome. Patient evaluation will be conducted in an outpatient clinic and involve 3 visits. Visit 1 will involve the collection and registration of social demographic, clinical, and functional data. Visit 2 will entail polysomnography, bronchoscopy for the evaluation of tracheobronchomalacia, titration of the optimal pressure that reduces the degree of collapse of the airway, and electrical impedance tomography. In visit 3, patients exhibiting a reduction in collapse areas will be requested to undergo chest computed tomography during inspiration and forced expiration with and without positive pressure (titrated to determine optimal CPAP pressure).

Results: This protocol is a doctorate project. The project was submitted to the institutional review board on January 24, 2017, and approval was granted on February 2, 2017 (Brazilian Research database number CAAE 64001317.4.000.0068). Patient evaluations started in April 2018. Planned recruitment is based on volunteers' availability and clinical stability, and interventions will be conducted at least once a month to finish the project at the end of 2020. A preliminary analysis of each case will be performed after each intervention, but detailed results are expected to be reported in the first quarter of 2021.

Conclusions: There is no consensus on the best treatment options for managing Mounier-Kuhn syndrome. The use of positive pressure could maintain patency of the collapsed airways, functioning as a "pneumatic stent" to reduce the degree of airflow obstruction. This, in turn, could promote mobilization of thoracic secretion and improve pulmonary ventilation. 
Trial Registration: ClinicalTrails.gov NCT03101059; https://clinicaltrials.gov/ct2/show/NCT03101059.

International Registered Report Identifier (IRRID)： DERR1-10.2196/14786

(JMIR Res Protoc 2020;9(8):e14786) doi: 10.2196/14786

\section{KEYWORDS}

Mounier-Kuhn syndrome; trancheobronchomegaly; tracheobronchomalacia; positive pressure; bronchoscopy

\section{Introduction}

Mounier-Kuhn syndrome (MKS) or congenital tracheobronchomegaly is a chronic and rare airway morbidity associated with recurrent respiratory infections and characterized by dilation of the trachea and the main bronchi [1]. This disease was first clinically described by Mounier-Kuhn in 1932 [2]. The estimated prevalence of this syndrome in patients with pulmonary symptoms is between $0.4 \%$ and $1.6 \%$, and it mostly affects the male gender [1,3-5]. Diagnosis of the MKS is frequently made in the third or fourth decade of life when the symptoms are more exuberant [6]. Histological alterations seen in the disease can be used to explain structural defects such as tracheobronchomalacia (TBM), saccular diverticula between cartilages, and bulging and dilation of the trachea and bronchi $[1,7]$.

The predominant signs and symptoms of the disease include coughing, purulent and abundant expectoration, digital clubbing, dyspnea, snoring, wheezing, and recurrent respiratory infection [1]. The disease could be associated with other comorbidities such as gastroesophageal reflux disease, chronic obstructive pulmonary disease, bronchiectasis, and obstructive sleep apnea/hypopnea syndrome (OSAHS) [1,8-12].

Some of the observed symptoms could be a consequence of TBM occurrence in some patients, which is defined by more than $50 \%$ collapse of the intrathoracic trachea and bronchi [13-16]. The main clinical consequences of TBM are obstruction to expiratory airflow; secondary air trapping; decrease in the effectiveness of cough and bronchial hygiene; and facilitation of conditions that promote microorganism colonization, leading to recurrent respiratory infection $[4,13]$.

Despite the patient's normal pulmonary function, the occurrence of obstructive ventilatory disorder is not uncommon [1,17-19]. Thorax computed tomography (CT) shows dilatation of the trachea and the right and left main bronchi [4,20,21]. Tracheobronchial diverticula, bronchiectasis, air trapping, and emphysema are common findings, suggesting involvement of the small airways $[1,7,10,11,22-26]$. Bronchoscopy is the gold standard examination for TBM diagnosis [12,14-16,26].

Since TBM is a rare and poorly studied disease, there is no consensus on a specific therapy. Therapeutic management includes inhaled bronchodilators, corticosteroids, hypertonic solution, and mucolytic agents. Some complementary tools used for disease management include noninvasive positive respiratory pressure, vaccination, respiratory physiotherapy, and pulmonary rehabilitation [1,27-29]. Continuous positive airways pressure (CPAP) support has been reported as an option for the treatment of tracheomalacia $[5,15,30]$. The rationale for using positive pressure in TBM is to recover the patency of the collapsed airways, thereby reducing airflow obstruction and promoting sputum clearance.

Previous experience has shown that CPAP can reduce symptoms, infections, and morbidity/mortality caused by TBM [5,15,31-33]. Additionally, it is an effective therapy in patients diagnosed with concomitant OSAHS [34-36]. This study has two main objectives. First, we aim to confirm the presence of tracheal collapse and the main bronchi of patients with MKS through respiratory endoscopy. Second, we will attempt to optimize the pressure with the CPAP approach to reduce or reverse the pathological consequences of the syndrome on the ventilatory pattern. We also will investigate the prevalence of OSAHS in this population. We hypothesize that maintenance of greater patency of airways could improve pulmonary ventilation for a longer time, reduce symptoms, and improve the quality of life of these patients.

\section{Methods}

\section{Overview}

This is an interventional, open-label, single-arm clinical trial to be conducted on patients diagnosed with MKS, who are followed up in the outpatient clinic from a tertiary university hospital. Eligible patients diagnosed with MKS who are followed up and agree to participate in the study will be requested to sign an informed consent form after verbal explanation and clarification of the study are provided by the investigator.

The following patients will be excluded from the study: those who are contraindicated for bronchoscopy (with thrombocytopenia, incorrigible coagulopathies, refractory hypoxemia, recent acute myocardial infarction, unstable angina, acute cardiac arrhythmias, and refractory bronchospasm), pregnant women under the age of 18 years, and those suspected of having or diagnosed with mycobacteriosis or other related risk factors by the clinical investigator. The study is approved by the institution's ethical review board and funded by the Division of Pulmonology Obstruction Group.

\section{Study Design}

The study will be conducted in three steps including three patient visits. During visit 1 (day 1), sociodemographic information, modified Medical Research Council dyspnea questionnaire measures [37-39], the Saint Georges Respiratory questionnaire measures $[40,41]$ for health-related quality of life, and most recent spirometry values will be recorded. In addition, all eligible patients will receive $40 \mathrm{mg} /$ day oral prednisone for 7 days before endoscopy, to reduce pulmonary secretion and possible airways hyperreactivity. If the patient presents signs or symptoms that characterize an acute pulmonary infection, 
the inclusion will be postponed, and specific treatment will be prescribed to reschedule the inclusion.

During visit 2 (day $7 \pm 3$ days from visit 1 ), polysomnography and respiratory endoscopy will be performed. Full-night polysomnography will be performed in accordance with the recommendations of the American Academy of Sleep Medicine $[42,43]$.

After polysomnography, a strap of 32 self-adhesive electrodes strap will be attached at the level of fourth intercostal space around the chest, and patients' electrical impedance tomography data will be recorded concomitantly with bronchoscopy. Bronchoscopy will be performed in two stages: (1) sedation, analgesia, and evaluation of the presence of TBM followed by bronchial hygiene and (2) optimal pressure titrations to decrease airway collapse and EIT performance. The procedure sequence will be conducted in two stages, as described below.

\section{Stage 1}

The patient will be placed in a dorsal position for cardiac monitoring, noninvasive blood pressure measurement, and pulse oximetry. The nasal fossae will be lubricated with $2 \%$ topical lidocaine gel, followed by the introduction of nasopharyngeal catheter number 08 to provide supplemental oxygen. Fentanyl $(0.5-2 \mu \mathrm{g} / \mathrm{kg})$ and midazolam $(0.03-0.05 \mu \mathrm{g} / \mathrm{kg})$ will be administered intravenously to achieve light sedation. Propofol bolus (20-40 mg/dose) may be used to achieve the desired sedation. Next, the custom nasal mask of CPAP will be attached to the patient's face, allowing the passage of the video-bronchoscope (Standard Q180 Olympus) with an external diameter of $4.9 \mathrm{~mm}$ and a working channel of $2.0 \mathrm{~mm}$. Topical lidocaine at a dose of $1 \%$ without a vasoconstrictor (maximum dose of $7 \mathrm{mg} / \mathrm{kg}$ ) will be instilled in the larynx and tracheobronchial tree. This will be followed by secretion aspiration. Subsequently, the presence of fixed or dynamic tracheal and bronchial collapse during the respiratory cycle will be analyzed and recorded. Direct observational assessment will be defined at the site with the greatest tracheal collapse and main bronchi during inspiratory and expiratory normal ventilation. Patients who do not present significant airway collapse will be excluded from the next step.

\section{Stage 2}

To evaluate the reduction of airway collapse, the bronchoscope will be fixed with the aid of a customized catheter in the tracheal region at least $2 \mathrm{~cm}$ before the area of the greatest collapse, previously defined by three bronchoscopists present in the examination room. The CPAP titration will start with a pressure of $0 \mathrm{~cm} \mathrm{H} 2 \mathrm{O}$ and be gradually increased in steps by $2 \mathrm{~cm} \mathrm{H} 2 \mathrm{O}$ every 10 complete respiratory cycles until a pressure of $18 \mathrm{~cm}$ $\mathrm{H} 2 \mathrm{O}$ is reached (Figure 1).

Figure 1. Bronchoscopic view of the trachea and the catheter (blue). Trachea during $0 \mathrm{~cm} \mathrm{H} 2 \mathrm{O}$ pressure during inspiration (A) and during expiration (B). Trachea in $18 \mathrm{~cm} \mathrm{H}_{2} \mathrm{O}$ pressure during inspiration (C) and expiration (D)

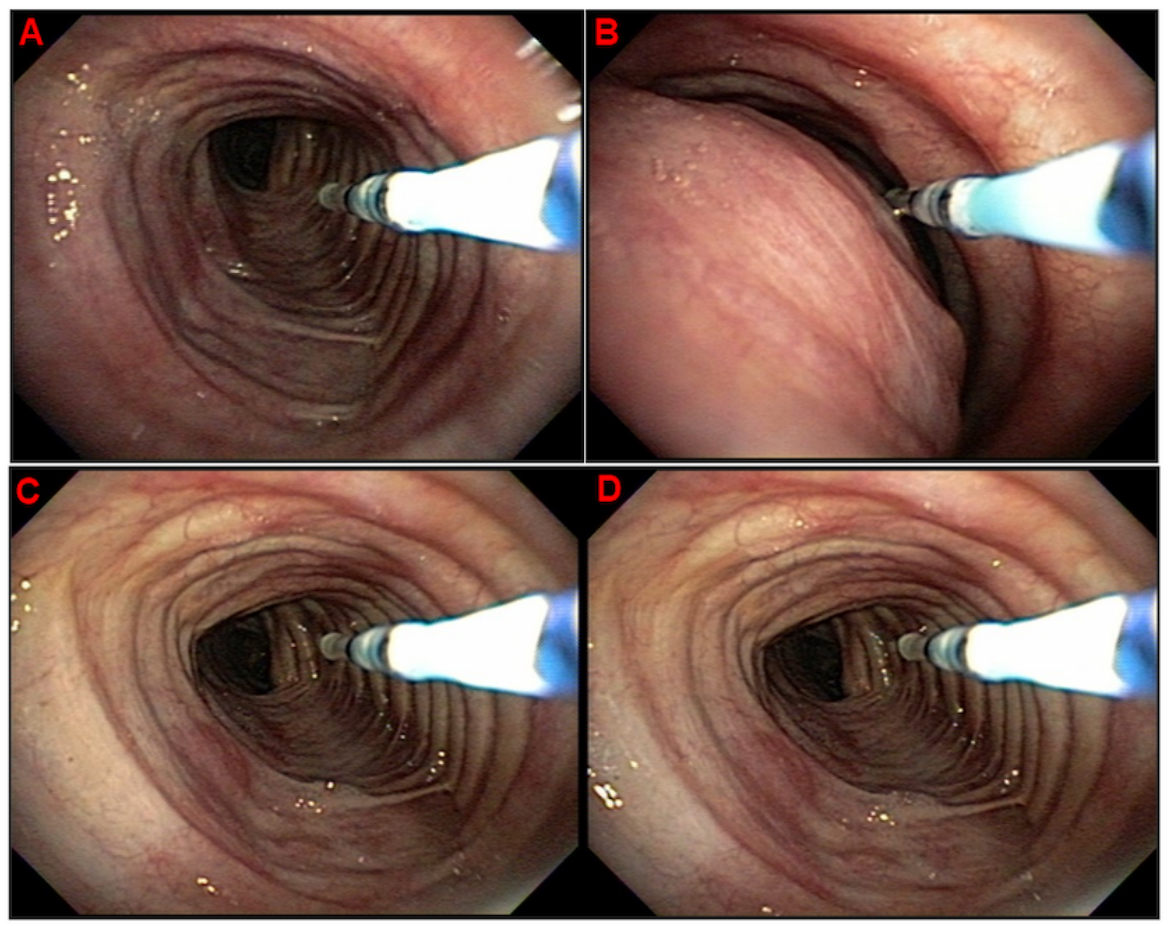

Through observational analysis and after a complete consensus among the bronchoscopists is reached, the minor pressure capable of reducing the degree of collapse in the trachea will be defined (P1). This procedure will be repeated in the main bronchus presenting major collapse. The entire procedure will be documented through video recording, and the images will be analyzed with software assistance (Image Processing
Toolbox, Matlab). Measures in the airway variation area will be analyzed by comparing the area of collapse at $0 \mathrm{~cm} \mathrm{H} 2 \mathrm{O}$ pressure with the corresponding measurements at different applied pressures titrated during the procedure. This analysis will allow us to find the pressure that reduces the degree of airway collapse (P2) $[42,43]$. EIT will be used for impact analysis of different pressures on pulmonary ventilation (distal 
airways), a functional imaging method that uses low-intensity electric currents. EIT can dynamically evaluate regional pulmonary ventilation by analyzing impedance variations and minimum impedance in a given thoracic segment by reproducing them in two-dimensional images. It is a noninvasive method that does not use ionizing radiation [44-47].

The EIT data will be acquired using ENLIGHT (Timpel), which produces 50 images per second, sampled in real time [44-47]. Distribution of ventilation will be analyzed at each CPAP level during the positive and expiratory pressure (PEEP) titration maneuver by dividing the EIT image into 4 quadrants (regions of interest, ROI), 2 of which will be gravity dependent (lower lobes) and 2 will be gravity independent (upper lobes). In patients who present tracheomalacia during bronchoscopy, a chest $\mathrm{CT}$ will be performed at visit 3 during inspiration and forced expiration in the presence or absence of CPAP. The ideal pressure for this will be obtained during bronchoscopy with an aim to evaluate and document the reduction of tracheal collapse. The images will be obtained by multislice tomography. No intravenous contrast will be administrated.

\section{Statistical Analysis}

Because MKS is a rare disease, there will be no sample size calculation. It will be a convenience sample including all eligible patients from Mounier-Kuhn reference centers in Brazil. As of July 2020, we are following at least 15 patients with MKS.

We will perform five main types of descriptive analysis on the data: (1) sociodemographic data of all MKS populations included in the study; (2) comparative analysis of the clinical-functional data of patients with and without collapse; (3) prevalence of sleep apnea/hypopnea syndrome; (4) repeated measurement techniques (bidirectional analysis of variance and Tukey or Sidak post hoc test when there is a difference) correlating the pressure that reverses the collapse (P1 and P2), to analyze the difference between airway collapse that occurs with and without PEEP; and (5) comparisons in the distribution of EIT ventilation with and without PEEP. Statistical analysis will be performed with statistical packages Sigma Stat V3 or SPSS V22, and an $\alpha$ level $\leq .05$ will be considered significant for all tests.

\section{Results}

The project was submitted to the institutional review board on January 24, 2017, and approved on February 2, 2017 (Brazilian Research database number CAAE 64001317.4.000.0068). The project was registered in ClinicalTrails.gov (NCT03101059) on March 23, 2017. This protocol is a doctorate project. Patient evaluations started in April 2018. Planned recruitment is based on volunteers' availability and clinical stability, and the interventions will be conducted at least once a month and completed at the end of 2020. A preliminary analysis of each case will be performed after each intervention, but detailed results are expected to be closed in the first quarter of 2021. We plan to publish 3 papers on this project. The proposed schedule for publication of the papers is as follows: the intervention protocol in 2019-2020; literature review about the concept of tracheobronchomalacia and airway collapse according to diverse uses ways to measure them and their definition in 2020; and results of the presence of tracheal collapse and the main bronchi of patients with MKS, through respiratory endoscopy and optimum positive pressure with CPAP that reduces or reverse it, in 2021 .

\section{Discussion}

Thus far, the main finding of this study is the identification of the progressive increase in the degree of pressure that can reverse the collapse of the trachea and bronchi and stabilize the airway.

MKS or congenital TBM is a rare and poorly studied disease. It has a wide spectrum of signs and symptoms and can have a great impact on the quality of life. Dyspnea, accumulation of secretions in the airways, bronchiectasis, and recurrent respiratory infections increase the morbimortality of the disease.

There is no consensus on the best treatment. In general, measures extrapolated from other pulmonary pathologies, such as those employed in chronic obstructive pulmonary disease and bronchiectasis, are used for therapeutic management. Among the specific treatments currently available, we want to highlight the endotracheal and endobronchial prosthesis and tracheobroncoplasty surgery. However, both approaches have limited results and are associated with frequent complications.

There is a rationale for the use of positive pressure. The use of positive pressure could maintain the patency of the collapsed airways, thereby providing a "pneumatic stent," which in turn could reduce airflow obstruction and promote mobilization of secretions within the airways. However, no study protocol has been performed to test this hypothesis.

The goal of this study is to assess if positive pressure with CPAP can be applied to reduce the extent of airway collapse and improve ventilation in MKS patients with tracheobronchomalacia. At the same time, we will evaluate the prevalence of OSAHS, a related and frequently occurring comorbidity that contributes to the worsening quality of life of patients with MKS.

\section{Acknowledgments}

This study is funded by the Division of Pulmonology Obstruction Group. The funding body will not interfere in the study design, data collection, data analysis, and interpretation of data, and writing of the manuscript.

\section{Conflicts of Interest}

None declared. 


\section{References}

1. Krustins E, Kravale Z, Buls A. Mounier-Kuhn syndrome or congenital tracheobronchomegaly: A literature review. Respiratory Medicine 2013 Dec;107(12):1822-1828. [doi: 10.1016/j.rmed.2013.08.042] [Medline: 24070565]

2. Mounier-Kuhn P. Dilatation de la trachee: constatations radiographiques et bronchoscopiques. Lyon Med 1932;150:106-109.

3. Katz I, Levine M, Herman P. Tracheobronchomegaly. The Mounier-Kuhn syndrome. Am J Roentgenol Radium Ther Nucl Med Dec 1962:88-94. [Medline: $\underline{13958486}$ ]

4. Özdemir C, Sökücü SN, Karasulu L, Altın S, Dalar L. Tracheomalacia Treatment Using a Large-Diameter, Custom-Made Airway Stent in a Case with Mounier-Kuhn Syndrome. Case Reports in Pulmonology 2014;2014:1-4. [doi: $10.1155 / 2014 / 910135]$

5. Adliff M, Ngato D, Keshavjee S, Brenaman S, Granton JT. Treatment of Diffuse Tracheomalacia Secondary to Relapsing Polychondritis With Continuous Positive Airway Pressure. Chest 1997 Dec;112(6):1701-1704. [doi: 10.1378/chest.112.6.1701]

6. Geppert EF. Recurrent Pneumonia. Chest 1990 Sep;98(3):739-745. [doi: 10.1378/chest.98.3.739]

7. Van Schoor J, Joos G, Pauwels R. Tracheobronchomegaly--the Mounier-Kuhn syndrome: report of two cases and review of the literature. Eur Respir J 1991 Nov;4(10):1303-1306. [Medline: 1804681]

8. Odell DD, Shah A, Gangadharan SP, Majid A, Michaud G, Herth F, et al. Airway Stenting and Tracheobronchoplasty Improve Respiratory Symptoms in Mounier-Kuhn Syndrome. Chest 2011 Oct;140(4):867-873. [doi: 10.1378/chest.10-2010]

9. Votto JJ, Trueb D, McCormick JR. Sleep apnea contributing to respiratory failure in the Mounier-Kuhn syndrome. Conn Med 1985 Feb;49(2):73-76. [Medline: $\underline{\text { 3971720] }}$

10. Roditi G, Weir J. The association of tracheomegaly and bronchiectasis. Clinical Radiology 1994 Sep;49(9):608-611. [doi: 10.1016/s0009-9260(05)81876-8]

11. Cartier Y, Kavanagh PV, Johkoh T, Mason AC, Müller NL. Bronchiectasis: accuracy of high-resolution CT in the differentiation of specific diseases. American Journal of Roentgenology 1999 Jul;173(1):47-52. [doi: 10.2214/ajr.173.1.10397098]

12. Sundaram P, Joshi JM. Tracheobronchomegaly associated tracheomalacia: analysis by sleep study. Indian J Chest Dis Allied Sci 2004;46(1):47-49. [Medline: 14870869]

13. Carden KA, Boiselle PM, Waltz DA, Ernst A. Tracheomalacia and Tracheobronchomalacia in Children and Adults. Chest 2005 Mar;127(3):984-1005. [doi: 10.1378/chest.127.3.984]

14. Jiang A, Gao X, Lu H. Diagnosis and management of an elderly patient with severe tracheomalacia: A case report andreview of the literature. Exp Ther Med Sep;. Epub013 Jul 2013;6(3):2. [doi: 10.3892/etm.2013.1195] [Medline: 4137262 ]

15. Murgu S, Colt H. Treatment of adult tracheobronchomalacia and excessive dynamic airway collapse : an update. Treat Respir Med 2006;5(2):103-115. [doi: 10.2165/00151829-200605020-00004] [Medline: 16512691]

16. Murgu S, Colt H. Tracheobronchomalacia and Excessive Dynamic Airway Collapse. Clinics in Chest Medicine 2013 Sep;34(3):527-555. [doi: 10.1016/j.ccm.2013.05.003]

17. Benesch M, Eber E, Pfleger A, Zach M. Recurrent lower respiratory tract infections in a 14-year-old boy with tracheobronchomegaly (Mounier-Kuhn syndrome). Pediatr. Pulmonol 2000 Jun;29(6):476-479. [doi: 10.1002/(sici)1099-0496(200006)29:6<476::aid-ppul10>3.0.co;2-r]

18. Pilavaki M, Anastasiadou K, Vlachojanni E, Drosos H, Petridis A, Drevelengas A. [Tracheobronchomegaly (Mounier-Kuhn syndrome): roentgen findings and tracheal stent instrumentation]. Pneumologie 1995 Oct;49(10):556-558. [Medline: 8584526]

19. Braham W, Daboussi S, Darouaz S, Ben Salem H, Boudawara N, Knani J. Trachéobronchomégalie avec exploration fonctionnelle respiratoire normale : une association rare. Revue de Pneumologie Clinique 2010 Dec;66(6):363-366. [doi: 10.1016/j.pneumo.2009.10.008]

20. Kamel KS, Lau G, Stringer MD. In vivo and in vitro morphometry of the human trachea. Clin. Anat 2009 Jul;22(5):571-579. [doi: 10.1002/ca.20815]

21. Kumar S, Mittal AK. Mounier-Kuhn syndrome (MKS) - Pathognomonic Findings. J Clin Diagn Res 2014 Dec;8(12):RJ01-RJ02 [FREE Full text] [doi: 10.7860/JCDR/2014/10829.5325] [Medline: 25654012]

22. Celik B, Bilgin S, Yuksel C. Mounier-Kuhn syndrome: a rare cause of bronchial dilation. Tex Heart Inst J 2011;38(2):194-196 [FREE Full text] [Medline: 21494536]

23. Schwartz M, Rossoff L. Tracheobronchomegaly. Chest 1994 Nov;106(5):1589-1590. [doi: 10.1378/chest.106.5.1589]

24. Himalstein MR, Gallagher JC. Tracheobronchiomegaly. Ann Otol Rhinol Laryngol 2016 Jun 29;82(2):223-227. [doi: 10.1177/000348947308200222]

25. Kent BD, Sulaiman I, Akasheh NB, Nadarajan P, Moloney E, Lane SJ. An unusual cause of recurrent spontaneous pneumothorax: the Mounier-Kuhn syndrome. Ir Med J 2011 May;104(5):152-153. [Medline: 21736094]

26. Westerlaan HE, Willems TP, Oudkerk M. Tracheobronchomegaly with bullous emphysema. JBR-BTR 2006;89(3):164-165. [Medline: 16883774]

27. Fortuna FP, Irion K, Wink C, Boemo JL. Síndrome de Mounier-Kühn. J. bras. pneumol 2006 Apr;32(2):180-183. [doi: 10.1590/s1806-37132006000200015] 
28. Collard P, Freitag L, Reynaert MS, Rodenstein DO, Francis C. Respiratory failure due to tracheobronchomalacia. Thorax 1996 Feb 01;51(2):224-226. [doi: 10.1136/thx.51.2.224]

29. Randak CO, Weinberger M. A child with progressive multiple tracheal diverticulae: A variation of the Mounier-Kuhn syndrome. Pediatr. Pulmonol 2012 Sep 04;48(8):841-843. [doi: 10.1002/ppul.22663]

30. Drain AJ, Perrin F, Tasker A, Stewart S, Wells F, Tsui S, et al. Double lung transplantation in a patient with tracheobronchomegaly (Mounier-Kuhn syndrome). J Heart Lung Transplant 2006 Jan;25(1):134-136. [doi: 10.1016/j.healun.2005.06.018] [Medline: 16399544]

31. Colt H, Murgu S. CPAP Treatment for Moderate Diffuse Excessive Dynamic Airway Collapse Caused by Mounier-Kuhn Syndrome. In: Bronchoscopy and Central Airway Disorders A Patient-Centered Approach. 1 edition. USA: Elsevier; 2012.

32. Wiseman NE, Duncan PG, Cameron CB. Management of tracheobronchomalacia with continuous positive airway pressure. Journal of Pediatric Surgery 1985 Oct;20(5):489-493. [doi: 10.1016/s0022-3468(85)80471-1]

33. Ferguson GT, Benoist J. Nasal Continuous Positive Airway Pressure in the Treatment of Tracheobronchomalacia. Am Rev Respir Dis 1993 Feb;147(2):457-461. [doi: 10.1164/ajrccm/147.2.457]

34. Spicuzza L, Caruso D, Di Maria G. Obstructive sleep apnoea syndrome and its management. Therapeutic Advances in Chronic Disease 2015 Jul 09;6(5):273-285. [doi: 10.1177/2040622315590318]

35. Strohl, M.D. KP, Baskin, M.D. J, Lance, M.D. C, Ponsky, M.D. D, Weidenbecher, M.D. M, Strohl, B.A. M, et al. Origins of and implementation concepts for upper airway stimulation therapy for obstructive sleep apnea. Respiratory Investigation 2016 Jul;54(4):241-249. [doi: 10.1016/j.resinv.2016.01.006]

36. Strohl KP, Redline S. Nasal CPAP therapy, upper airway muscle activation, and obstructive sleep apnea. Am Rev Respir Dis 1986 Sep;134(3):555-558. [doi: 10.1164/arrd.1986.134.3.555] [Medline: $\underline{3530073]}$

37. Global Strategy for the Diagnosis, Management and prevention of Chronic, Obstructive Pulmonary Disease, 2018 Report; 2017. GOLD COPD. URL: http://goldcopd.org/ [accessed 2018-07-01]

38. Bestall JC, Paul EA, Garrod R, Garnham R, Jones PW, Wedzicha JA. Usefulness of the Medical Research Council (MRC) dyspnoea scale as a measure of disability in patients with chronic obstructive pulmonary disease. Thorax 1999 Jul 01;54(7):581-586. [doi: 10.1136/thx.54.7.581]

39. Mahler DA, Ward J, Waterman LA, McCusker C, ZuWallack R, Baird JC. Patient-Reported Dyspnea in COPD Reliability and Association With Stage of Disease. Chest 2009 Dec;136(6):1473-1479. [doi: 10.1378/chest.09-0934]

40. Guyatt GH, Berman LB, Townsend M, Pugsley SO, Chambers LW. A measure of quality of life for clinical trials in chronic lung disease. Thorax 1987 Oct 01;42(10):773-778. [doi: 10.1136/thx.42.10.773]

41. Jones PW, Quirk FH, Baveystock CM, Littlejohns P. A Self-complete Measure of Health Status for Chronic Airflow Limitation: The St. George's Respiratory Questionnaire. Am Rev Respir Dis 1992 Jun;145(6):1321-1327. [doi: 10.1164/ajrccm/145.6.1321]

42. Genta P, Schorr F, Eckert D, Gebrim E, Kayamori F, Moriya H, et al. Upper airway collapsibility is associated with obesity and hyoid position. Sleep 2014 Oct 01;37(10):1673-1678 [FREE Full text] [doi: 10.5665/sleep.4078] [Medline: 25197805]

43. Genta P, Edwards B, Sands S, Owens R, Butler J, Loring S, et al. Tube Law of the Pharyngeal Airway in Sleeping Patients with Obstructive Sleep Apnea. Sleep 2016 Feb 01;39(2):337-343 [FREE Full text] [doi: 10.5665/sleep.5440] [Medline: 26446124]

44. Barber D, Brown B. Applied potential tomography. J Br Interplanet Soc 1989 Aug;42(7):391-393. [Medline: 11540235]

45. Frerichs I, Hahn G, Schiffmann H, Berger C, Hellige G. Monitoring regional lung ventilation by functional electrical impedance tomography during assisted ventilation. Ann N Y Acad Sci 1999 Apr 20;873(1 ELECTRICAL BI):493-505. [doi: 10.1111/j.1749-6632.1999.tb09498.x] [Medline: 10372185]

46. Victorino JA, Borges JB, Okamoto VN, Matos GFJ, Tucci MR, Caramez MPR, et al. Imbalances in Regional Lung Ventilation. Am J Respir Crit Care Med 2004 Apr;169(7):791-800. [doi: 10.1164/rccm.200301-133oc]

47. Costa EL, Lima RG, Amato MB. Electrical impedance tomography. Current Opinion in Critical Care 2009;15(1):18-24. [doi: $10.1097 / \mathrm{mcc} .0 \mathrm{~b} 013 \mathrm{e} 3283220 \mathrm{e} 8 \mathrm{c}]$

\section{Abbreviations}

CPAP: continuous positive airway pressure

CT: computed tomography

EIT: electrical impedance tomography

MKS: Mounier-Kuhn syndrome

OSAHS: obstructive sleep apnea-hypopnea syndrome

TBM: tracheobronchomalacia 
Edited by $G$ Eysenbach; submitted 22.05.19; peer-reviewed by $G$ Segrelles-Calvo, D Peña; comments to author 24.03.20; revised version received 28.04.20; accepted 02.06.20; published 14.08.20

Please cite as:

Lima E, Nakamura MAM, Genta PR, Rodrigues AJ, Athanazio RA, Rached S, Costa ELV, Stelmach R

Improving Airways Patency and Ventilation Through Optimal Positive Pressure Identified by Noninvasive Mechanical Ventilation

Titration in Mounier-Kuhn Syndrome: Protocol for an Interventional, Open-Label, Single-Arm Clinical Trial

JMIR Res Protoc 2020;9(8):e14786

URL: http://www.researchprotocols.org/2020/8/e14786/

doi: $\underline{10.2196 / 14786}$

PMID: $\underline{32795996}$

CEvelise Lima, Maria Aparecida Miyuki Nakamura, Pedro Rodrigues Genta, Ascedio José Rodrigues, Rodrigo Abensur Athanazio, Samia Rached, Eduardo Leite Vieira Costa, Rafael Stelmach. Originally published in JMIR Research Protocols (http://www.researchprotocols.org), 14.08.2020. This is an open-access article distributed under the terms of the Creative Commons Attribution License (https://creativecommons.org/licenses/by/4.0/), which permits unrestricted use, distribution, and reproduction in any medium, provided the original work, first published in JMIR Research Protocols, is properly cited. The complete bibliographic information, a link to the original publication on http://www.researchprotocols.org, as well as this copyright and license information must be included. 